\title{
Local Stabilization of Discrete-Time T-S Fuzzy Systems with Magnitude- and Energy-Bounded Disturbances
}

\author{
Donghwan Lee ${ }^{\mathrm{a}}$, Jianghai $\mathrm{Hu}^{\mathrm{a}}$ \\ ${ }^{a}$ Department of Electrical and Computer Engineering, Purdue University, West Lafayette, IN 47906, USA
}

\begin{abstract}
Recently, the local stability and local stabilization problems have been investigated by several authors for nonlinear systems described by Takagi-Sugeno (T-S) fuzzy systems. In this paper, we study the local stabilization problem for discrete-time T-S fuzzy systems with magnitude- and energy-bounded disturbances. The control design problem is cast as a linear matrix inequality (LMI) optimization. The state-feedback controller is designed in such a way that, if the initial state is within a certain domain, then the state trajectories under bounded disturbances will not escape the fuzzy modelling region of the state space. Finally, examples are given to demonstrate the validity of the proposed method.
\end{abstract}

Key words: Nonlinear systems; Takagi-Sugeno (T-S) fuzzy systems; Lyapunov stability; linear matrix inequality (LMI); robust control.

\section{Introduction}

The Takagi-Sugeno (T-S) fuzzy systems are a class of systems modelling where nonlinear systems can be described as a convex combination of linear subsystems weighted by the nonlinear membership functions (MFs). The T-S fuzzy systems have received much attention during the last decades [1, 5, 11, 19, 23, 32, 34, 35, 39], because they can provide a systematic framework to take advantage of linear system theories for stability analysis and control design problems of nonlinear systems. Especially, if the Lyapunov stability theory is used for the T-S fuzzy model, several control design problems can be formulated as convex linear matrix inequality (LMI) optimization procedures easily solvable via standard convex optimization techniques [3].

The simplest Lyapunov method is to use the common quadratic Lyapunov function. For instance, [38, 41] used the quadratic Lyapunov function to address the stability and stabilization problems of the T-S fuzzy systems. However, the common quadratic Lyapunov approach is known to entail a considerable conservatism in general, because a common Lyapunov function should be found for all subsystems of the fuzzy systems. During the last decade, efforts have been made to reduce the conservatism of the common quadratic Lyapunov approach. A natural way is to construct generalized Lyapunov functions, for instance, the piecewise Lyapunov functions [8, 12], fuzzy Lyapunov functions [7, 9, 10, 24, 36, 37, 43], a class of Lyapunov functions using line-integral [28]; and polynomial Lyapunov functions $[16,31,40]$. Besides, other directions of reducing the conservatism are approaches based on MFs' shape [15, 25, 30] and Pólya's theorem [29].

Recently, the notion of the local stability and local stabilization approaches were investigate in $[2,17,18,26]$. Not only can these approaches reduce the conservatism of the non-local (global) approaches, but they can be also used to establish more rigorous stability analysis and control design procedures for the T-S fuzzy systems. Roughly speaking, the local stability/stabilization problems are determining if there exists a neighborhood of the equilibrium point, called the domain of attraction (DA) [14], such that all trajectories of the system emanating from any initial point in the DA asymptotically converges to the equilibrium point. Especially for T-S fuzzy systems, the local analysis and design are important in the sense that the T-S fuzzy model is only valid when the state is inside a local modelling

Email addresses: lee1923@purdue.edu (Donghwan Lee), jianghai@purdue .edu (Jianghai Hu) 
region, denoted by $\mathcal{L}$, of the $\mathrm{T}-\mathrm{S}$ fuzzy systems. If the system under consideration turns out to be locally stable, then another important problem is to estimate the DA. For the T-S fuzzy systems, the local stability and the DA analysis can be used to guarantee that all the trajectories under a certain condition do not escape region $\mathcal{L}$, and thus ensure the asymptotic stability of the open-loop or closed-loop system. Compared to the local stability approaches $[2,26]$ for the continuous-time T-S fuzzy systems, not until recently has the discrete-time counterpart been studied in [17, 18], where the notion of the one-step invariant set and the set of the bounded variation rates of the MFs were introduced. The one-step invariant set, $\mathcal{R}$, represents a subset of the fuzzy modelling region $\mathcal{L}$ whose next state along the state trajectories does not escape $\mathcal{L}$. The set of the bounded variation rates of the MFs, denoted by $\mathcal{H}(b)$, is a subset of the modelling region $\mathcal{L}$, where the variation rates of the MFs' values along the state trajectories inside the set are bounded above by the constant $b \in(0,1]$. Using these concepts, it was proved in [17] that a sublevel set of the Lyapunov function confined within the intersection of the two sets, i.e., $\mathcal{R} \cap \mathcal{H}(b)$, is an estimation of the DA. In addition, convex computational methods based on LMIs were developed in $[17,18]$ to find the Lyapunov functions and to estimate the DA.

More recently, the local stabilization problems were studied in [42] for continuous-time T-S fuzzy systems under the assumption that the system is subject to the magnitude- and energy-bounded disturbances. In the paper [42], LMI-based design procedures were developed, and it was proved that the resulting closed-loop system guarantees that any state starting from some region does not escape the modelling region of the T-S fuzzy systems even if the system is affected by external disturbances. Although [42] have produced progress in the local stabilization problem under disturbances, to the authors' knowledge, the problem has not been investigated for the discrete-time T-S fuzzy systems so far.

Motivated by the above discussions, the goal of this paper is to extend the results in [42] to the local control design problem of the discrete-time T-S fuzzy systems under the magnitude- and energy-bounded disturbances. The main contributions of the paper can be summarized as follows: 1) We develop an LMI-based control design procedure for the local stabilization problem. Although the underlying assumptions are similar, the derivation procedures of the proposed design method and that in [42] are quite different. For instance, we consider the bounded variation rates of the MFs for the discrete-time systems by adopting the techniques developed in [17] to reduce the conservatism. In addition, we introduce the disturbance invariant set $\mathcal{R}$, which represents a subset of the modelling region $\mathcal{L}$ whose element (state vector) in the next time step does not escape $\mathcal{L}$ for all possible disturbances with the bounded magnitude and energy; 2) The proposed local stabilization approach can deal with the following two cases. If the disturbances exist, then we can compute a subset of the modelling region, which guarantees that for any initial state inside the subset, its all possible future trajectories will remain within the subset. Furthermore, we also prove that every initial states in the subset will converge to the origin, i.e., the local asymptotic stability of the closed-loop system. If there is no disturbance, then a lager inner estimation of the DA can be computed. 3) The design methods in [42] include additional design parameters that should be predetermined by the designer, while the design scheme in this paper reduces the search space by linearizing some bilinear terms. This simplifies the design procedure. The developed design method is formulated as a single-parameter minimization problem subject to LMIs, which can be efficiently solved via a sequence of LMI optimizations, such as a bisection algorithm or a line search process. Finally, illustrative examples are given to show the effectiveness of the proposed approach.

\section{Preliminaries}

\subsection{Notation}

The adopted notation is as follows: $\mathbb{R}$ and $\mathbb{Z}_{+}$: sets of real numbers and nonnegative integers, respectively; $A^{T}$ : transpose of matrix $A ; A>0(A<0, A \geq 0$, and $A \leq 0$, respectively): symmetric positive definite (negative definite, positive semi-definite, and negative semi-definite, respectively) matrix $A ; I_{n}$ and $0_{n \times m}: n \times n$ identity matrix and $n \times m$ zero matrix, respectively; $0_{n}$ : origin of $\mathbb{R}^{n} ; \mathrm{He}\{A\}$ : short hand notion for $A^{T}+A ; \operatorname{co}\{\cdot\}$ : convex hull [3]; $\partial \mathcal{S}$ : boundary of set $\mathcal{S} ; *$ inside a matrix: transpose of its symmetric term; $\mathcal{I}_{N}:=\{1,2, \ldots, N\} ; \operatorname{diag}\{A, B\}$ : block diagonal matrix with blocks $A$ and $B$. Throughout the paper, the following shorthand is used for ease of notation:

$$
\begin{aligned}
& \text { - } \alpha(z(k)):=\left[\begin{array}{llll}
\alpha_{1}(z(k)) & \alpha_{2}(z(k)) & \cdots & \alpha_{N}(z(k))
\end{array}\right]^{T} \in \mathbb{R}^{N} \\
& \text { - } \Delta \alpha_{i}(z(k)):=\alpha_{i}(z(k+1))-\alpha_{i}(z(k))
\end{aligned}
$$


- $\Delta \alpha(z(k)):=\alpha(z(k+1))-\alpha(z(k)) \in \mathbb{R}^{N}$

- $\Upsilon(\alpha):=\sum_{i=1}^{N} \alpha_{i}(z(k)) \Upsilon_{i}$

- $\Upsilon(\alpha+\Delta \alpha):=\sum_{i=1}^{N} \alpha_{i}(z(k+1)) \Upsilon_{i}$

Given any function $f: \mathbb{R}^{p} \rightarrow \mathbb{R}$,

$$
\frac{\partial f(z)}{\partial z}:=\left[\begin{array}{llll}
\partial f(z) / \partial z_{1} & \partial f(z) / \partial z_{2} & \cdots & \partial f(z) / \partial z_{p}
\end{array}\right] .
$$

Through the paper, it is assumed that the gradient is always a row vector.

\subsection{Problem formulation}

Consider the discrete-time nonlinear system

$$
x(k+1)=f(x(k), u(k), w(k)),
$$

where $k \in \mathbb{Z}_{+}, x(k):=\left[\begin{array}{lll}x_{1}(k) & \cdots & x_{n}(k)\end{array}\right]^{T} \in \mathbb{R}^{n}$ is the state, $u(k) \in \mathbb{R}^{m}$ is the control input, $w(k) \in \mathbb{R}^{q}$ is the external disturbance, $f: \mathbb{R}^{n} \times \mathbb{R}^{m} \times \mathbb{R}^{q} \rightarrow \mathbb{R}^{n}$ is a nonlinear function such that $f\left(0_{n}, 0_{m}, 0_{q}\right)=0_{n}$, i.e., the origin is an equilibrium point of (1). By the sector nonlinearity approach [39], there exists a class of the nonlinear systems which can be exactly represented by the T-S fuzzy system

$$
x(k+1)=\sum_{i=1}^{N} \alpha_{i}(z(k))\left(A_{i} x(k)+B_{i} u(k)+B_{w i} w(k)\right), \quad \forall x(k) \in \mathcal{L},
$$

where $k \in \mathbb{Z}_{+}, \mathcal{L} \in \mathbb{R}^{n}$ is a set of the state variables including the origin, $A_{i} \in \mathbb{R}^{n \times n}, B_{w i} \in \mathbb{R}^{q \times m}$ are constant matrices, $i \in \mathcal{I}_{N}:=\{1,2, \ldots, N\}$ is the rule number,

$$
z(k):=\left[\begin{array}{lll}
z_{1}(k) & \cdots & z_{p}(k)
\end{array}\right]^{T} \in \mathbb{R}^{p}
$$

is the vector containing premise variables in the fuzzy inference rule, $\alpha_{i}: \mathbb{R}^{p} \rightarrow[0,1]$ is the MF (membership function) for each rule $i \in \mathcal{I}_{N}$ such that the vector of the MFs

$$
\alpha(z(k)):=\left[\begin{array}{lll}
\alpha_{1}(z(k)) & \cdots & \alpha_{N}(z(k))
\end{array}\right]^{T} \in \mathbb{R}^{N}
$$

lies in the unit simplex $\Lambda_{N}$ for all $(k, x(k)) \in \mathbb{Z}_{+} \times \mathcal{L}$, where

$$
\Lambda_{N}:=\left\{\delta \in \mathbb{R}^{N}: \sum_{i=1}^{N} \delta_{i}=1, \quad 0 \leq \delta_{i} \leq 1, \quad i \in \mathcal{I}_{N}\right\} .
$$

Note that to obtain the T-S fuzzy system (2), the nonlinear system (1) should be affine in the input $u(k)$ and the disturbance $w(k)$. In this paper, we assume that the premise variables are linear combinations of the state variables:

$$
z(k)=\mathcal{T} x(k)=\left[\begin{array}{c}
\mathcal{T}_{1} \\
\vdots \\
\mathcal{T}_{p}
\end{array}\right] x(k) \in \mathbb{R}^{p}, \quad \mathcal{T} \in \mathbb{R}^{p \times n} .
$$

Under this assumption, $\mathcal{L} \subseteq \mathbb{R}^{n}$ can be represented by the set of state variables satisfying $\mathcal{L} \subseteq\left\{x \in \mathbb{R}^{n}\right.$ : $\left.f(x, u, w)=A(\alpha(z)) x+B(\alpha(z)) u+B_{w}(\alpha(z)) w, \alpha(z) \in \Lambda_{N}, z=\mathcal{T} x, u \in \mathbb{R}^{m}, w \in \mathbb{R}^{q}\right\}$. Moreover, the following assumptions will be considered.

Assumption 1. The modelling region $\mathcal{L}$ is described as

$$
\mathcal{L}:=\left\{x \in \mathbb{R}^{n}: \mathcal{T}_{l} x \in\left[-z_{l, \max }, z_{l, \max }\right], \quad \forall l \in \mathcal{I}_{p}\right\}
$$

where $z_{l, \max }>0, l \in \mathcal{I}_{p}$ are a priori given real numbers. 
Assumption 2. For each $i \in \mathcal{I}_{N}$, we can obtain the set of vertices $\mathcal{G}_{i}$ of the polytope that includes $\partial \alpha_{i}(\xi) / \partial \xi$ for all $\xi=\mathcal{T} x, x \in \mathcal{L}$.

Example 1. Consider the discrete-time nonlinear truck-trailer model given in [38]

$$
\left\{\begin{array}{c}
x_{1}(k+1)=\left(1-\frac{v T_{s}}{L}\right) x_{1}(k)+\frac{v T_{s}}{l} u(k)+w(k) \\
x_{2}(k+1)=\frac{v T_{s}}{L} x_{1}(k)+x_{2}(k) \\
x_{3}(k+1)=x_{3}(k)+v T_{s} \sin \left(x_{2}(k)+\frac{v T_{s}}{2 L} x_{1}(k)\right)
\end{array}\right.
$$

where $x_{1}(k)$ is the angle difference between the truck and the trailer, $x_{2}(k)$ is the angle of the trailer, $x_{3}(k)$ is the vertical position of the rear end of the trailer, $w(k)$ is the disturbance, $l$ is the length of the truck, $L$ is the length of the trailer, $T_{s}$ is the sampling time, and $v$ is the constant speed of the backward movement. To obtain its T-S fuzzy model, the premise variable is defined as $z_{1}(k)=x_{2}(k)+\frac{v T_{s}}{2 L} x_{1}(k)$. The system can be exactly recast as the discrete-time T-S fuzzy model (2) with

$$
\begin{aligned}
& A_{1}=\left[\begin{array}{ccc}
1-v T_{s} / L & 0 & 0 \\
v T_{s} / L & 1 & 0 \\
\left(v T_{s}\right)^{2} /(2 L) & v T_{s} & 1
\end{array}\right], \quad A_{2}=\left[\begin{array}{ccc}
1-v T_{s} / L & 0 & 0 \\
v T_{s} / L & 1 & 0 \\
\sigma\left(v T_{s}\right)^{2} /(2 L) & \sigma v T_{s} & 1
\end{array}\right], \quad \sigma=\frac{\sin z_{1, \max }}{z_{1, \max }}, \\
& B_{1}=B_{2}=\left[\begin{array}{c}
\frac{v T_{s}}{l} \\
0 \\
0
\end{array}\right], \quad B_{w 1}=B_{w 2}=\left[\begin{array}{c}
2 \\
0 \\
0
\end{array}\right], \quad z_{1}(k)=x_{2}(k)+\frac{v T_{s}}{2 L} x_{1}(k), \\
& \alpha_{1}\left(z_{1}(k)\right)=\left\{\begin{array}{l}
\frac{z_{1, \max } \sin z_{1}(k)-z_{1}(k) \sin z_{1, \max }}{z_{1}(k)\left(z_{1, \max }-\sin z_{1, \max }\right)}, \quad z_{1}(k) \neq 0 \\
1, \quad z_{1}(k)=0
\end{array}\right. \\
& \alpha_{2}\left(z_{1}(k)\right)=1-\alpha_{1}\left(z_{1}(k)\right)=\left\{\begin{array}{l}
\frac{z_{1, \max }\left(z_{1}(k)-\sin z_{1}(k)\right)}{z_{1}(k)\left(z_{1}, \max -\sin z_{1, \max }\right)} \\
0, \quad z_{1}(k)=0
\end{array}\right.
\end{aligned}
$$

which is modelled within $\mathcal{L}=\left\{\xi \in \mathbb{R}^{n}:\left[\begin{array}{lll}v T_{s} / 2 L & 1 & 0\end{array}\right] \xi \in\left[-z_{1, \max }, z_{1, \max }\right]\right\}$.

Remark 1. As we have shown in Example 1, there is a class of nonlinear systems (1) which can be exactly represented by the T-S fuzzy model within a region $\mathcal{L} \subseteq \mathbb{R}^{n}$ defined in (4). In general, even if the exact T-S fuzzy representation cannot be obtained, it is possible to compute a fuzzy model which can approximate the original nonlinear system (1) with arbitrarily exact accuracy by the universal approximater argument [44]. In this case, only approximate models of (1) can be obtained in some domain of the state space, i.e.,

$$
\mathcal{L} \subseteq\left\{x \in \mathbb{R}^{n}:\left\|f(x, u)-\sum_{i=1}^{N} \alpha_{i}(z)\left[A_{i} x+B_{i} u+B_{w i} w\right]\right\| \leq \varepsilon, \quad z=g(x), \quad u \in \mathbb{R}^{m}, \quad w \in \mathbb{R}^{q}\right\}
$$

with $\varepsilon>0$. To take into account the approximation error in our control design approach, we can employ the robust control design in [21]. Specifically, the modelling region (6) is represented by

$$
\begin{aligned}
& \mathcal{L} \subseteq\left\{x \in \mathbb{R}^{n}: f(x, u) \in \sum_{i=1}^{N} \alpha_{i}(z)\left[A_{i}+\Delta A_{i}\right] x+\sum_{i=1}^{N} \alpha_{i}(z)\left[B_{i}+\Delta B_{i}\right] u\right. \\
& \left.+\sum_{i=1}^{N} \alpha_{i}(z)\left[B_{w i}+\Delta B_{w i}\right] w, \quad z=g(x), \quad u \in \mathbb{R}^{m}, \quad w \in \mathbb{R}^{q}\right\},
\end{aligned}
$$

where $\Delta A_{i} \subset \mathbb{R}^{n \times n}, \Delta B_{i} \subset \mathbb{R}^{n \times m}$, and $\Delta B_{w i} \subset \mathbb{R}^{n \times q}$ are bounded sets representing the uncertainties. Then, the robust stability conditions in $[20,21]$ can be applied to design robust controllers against the approximate errors. In this paper, we will only focus on the class of nonlinear systems where an exact fuzzy model can be obtained.

Throughout the paper, the following assumption will be used. 
Assumption 3 (Magnitude- and energy-bounded disturbance [42]). The disturbance $w: \mathbb{Z}_{+} \rightarrow \mathbb{R}^{q}$ satisfies $w(k)^{T} w(k) \leq$ $\varepsilon_{1}, \forall k \in \mathbb{Z}_{+}$and $\sum_{0}^{\infty} w(k)^{T} w(k) \leq \varepsilon_{2}$.

The problem addressed in this paper can be stated as follows:

Problem 1 (Local stability analysis).

1. Determine a state-feedback control law $u(k)$ such that under $w(k) \equiv 0_{p}$, the zero equilibrium point of (2) is locally asymptotically stable. In addition, estimate an invariant subset of the domain of attraction (DA);

2. Under Assumption 3, obtain a region such that any trajectories starting from the region do not escape it for all $k \in \mathbb{Z}_{+}$.

\section{Main result}

We present LMI-based optimization procedures to solve Problem 1. For $P_{i}>0, i \in \mathcal{I}_{N}$, consider

$$
V(x(k)):=x(k)^{T}\left(\sum_{i=1}^{r} \alpha_{i}(z(k)) P_{i}\right)^{-1} x(k)=x(t)^{T} P(\alpha)^{-1} x(t)
$$

defined for all $x(k) \in \mathcal{L}$ as a candidate of Lyapunov functions. It can be proved that if $P_{i}>0, i \in \mathcal{I}_{N}$, then $V$ is a positive definite and radially unbounded function, i.e., $V(x) \rightarrow \infty$ as $\|x\| \rightarrow \infty$ [14, Chapter 4]. In addition, consider the non-parallel distributed compensation state-feedback controller developed in [10]:

$$
u(k)=\left(\sum_{i=1}^{r} \alpha_{i}(z(k)) F_{i}\right)\left(\sum_{i=1}^{r} \alpha_{i}(z(k)) P_{i}\right)^{-1} x(k)=F(\alpha) P(\alpha)^{-1} x(t), \quad \forall x(k) \in \mathcal{L} .
$$

By plugging the above state-feedback control law into (2), one gets the closed-loop system

$$
x(k+1)=\left(A(\alpha)+B(\alpha) F(\alpha) P(\alpha)^{-1}\right) x(k)+B_{w}(\alpha) w(k)=\left[A_{c l}(\alpha) \quad B_{w}(\alpha)\right]\left[\begin{array}{c}
x(k) \\
w(k)
\end{array}\right], \quad \forall x(k) \in \mathcal{L},
$$

where $A_{c l}(\alpha):=A(\alpha)+B(\alpha) F(\alpha) P(\alpha)^{-1}$. For the development, we need to define the following sets:

- $\mathcal{H}(b):=\left\{x \in \mathcal{L}:\left|\Delta \alpha_{i}(z)\right| \leq b, z=\mathcal{T} x, \forall i \in \mathcal{I}_{N}\right\}$

- $\Omega(\gamma):=\{x \in \mathcal{L}: V(x) \leq \gamma\}$

- $\mathcal{R}:=\left\{x \in \mathcal{L}:\left[A_{c l}(\alpha) \quad B_{w}(\alpha)\right]\left[\begin{array}{c}x \\ w\end{array}\right] \in \mathcal{L}, w \in \mathbb{R}^{q}, w^{T} w \leq \varepsilon_{1}\right\}$

- $\mathcal{V}(b)$ : set of vertices of hyper rectangle $\left\{v \in \mathbb{R}^{N}:-b \leq v_{i} \leq b, \forall i \in \mathcal{I}_{N}\right\}$;

- $\mathcal{G}_{i}$ : set of vertices of a polytope that includes $\partial \alpha_{i}(\xi) / \partial \xi$ for all $\xi=\mathcal{T} x, x \in \mathcal{L}$.

$\mathcal{H}(b)$ is a subset of the modelling region $\mathcal{L}$, where the variation rates of the MFs are less than or equal to $b \in(-, 1]$. $\Omega(\gamma)$ is the $\gamma$-level set of the Lyapunov function $V$ in the modelling region $\mathcal{L}$. The set $\mathcal{R}$ can be interpreted as a disturbance invariant set considered in [13], [27]. In other words, $\mathcal{R}$ is a subset of the modelling region $\mathcal{L}$ such that any state vector inside $\mathcal{R}$ will remain in $\mathcal{L}$ in the next time step along the state trajectories with any possible disturbance $w \in \mathbb{R}^{q}$ satisfying $w^{T} w \leq \varepsilon_{1}$. The following lemmas will play important roles for the development of the main result.

Lemma 1 ([41, Theorem 2.2]). Given symmetric matrices $\Upsilon_{i j},(i, j) \in \mathcal{I}_{N}^{2}, \sum_{i=1}^{N} \sum_{j=1}^{N} a_{i}(z(k)) a_{j}(z(k)) \Upsilon_{i j} \prec 0$ holds for all $x(k) \in \mathcal{L}$ if LMIs $\Upsilon_{i i}<0, \forall i \in \mathcal{I}_{N}$ and $(2 /(N-1)) \Upsilon_{i i}+\Upsilon_{i j}+\Upsilon_{j i}<0, i \neq j, \forall(i, j) \in \mathcal{I}_{N}^{2}$ are fulfilled. 
Lemma 2 (Mean value theorem in several variables [4]). Let $\mathcal{U} \in \mathbb{R}^{p}$ be a convex set, and suppose $f: \mathcal{U} \rightarrow \mathbb{R}$ is continuously differentiable. Then, for any $x, y \in \mathcal{U}$, there is a real number $c \in[0,1]$ such that

$$
f(y)-f(x)=\frac{\partial f((1-c) y+c x)}{\partial x}(y-x) .
$$

Lemma 3 ([6, Theorem 1]). Let $x$ and $y$ are positive real numbers. Then, $-y^{2} x^{-1} \leq-2 y+x$ is satisfied.

Proof. Since $x>0$, inequality $-(x-y)^{2} x^{-1} \leq 0$ holds. This leads to $-y^{2} x^{-1} \leq-2 y+x$.

We are now in position to establish LMI-based optimization procedure that solves Problem 1.

Problem 2. Let the parameters $\varepsilon_{1}>0, \varepsilon_{2}>1$, and $b \in(0,1]$ be given. Solve

$\min _{P_{i}, F_{i}, \beta, \lambda} \beta$ subject to

$\left[\begin{array}{cc}-P_{i} & * \\ \mathcal{T}_{j} P_{i} & -2 z_{j, \max }+\left(1+\lambda \varepsilon_{2}\right)\end{array}\right] \prec 0, \quad \forall(i, j) \in \mathcal{I}_{N} \times \mathcal{I}_{p}$

$\Upsilon_{i i}^{[1]}(g)<0, \quad \frac{2}{N-1} \Upsilon_{i i}^{[1]}(g)+\Upsilon_{i j}^{[1]}(g)+\Upsilon_{j i}^{[1]}(g) \prec 0, \quad \forall(i, j) \in\left\{(i, j) \in \mathcal{I}_{N} \times \mathcal{I}_{N}: i \neq j\right\}, \quad \forall g \in \mathcal{G}_{k}, \quad \forall k \in \mathcal{I}_{N}$,

$\Upsilon_{i i k}^{[2]} \prec 0, \quad \frac{2}{N-1} \Upsilon_{i i k}^{[2]}+\Upsilon_{i j k}^{[2]}+\Upsilon_{j i k}^{[2]} \prec 0, \quad \forall(i, j) \in\left\{(i, j) \in \mathcal{I}_{N} \times \mathcal{I}_{N}: i \neq j\right\}, \quad \forall k \in \mathcal{I}_{N}$,

$\left[\begin{array}{cc}-\beta I_{n} & * \\ I_{n} & -P_{i}\end{array}\right] \prec 0, \quad i \in \mathcal{I}_{N}$

$\Upsilon_{i i}^{[3]}(v)<0, \quad \frac{2}{N-1} \Upsilon_{i i}^{[3]}(v)+\Upsilon_{i j}^{[3]}(v)+\Upsilon_{j i}^{[3]}(v)<0, \quad \forall(i, j) \in\left\{(i, j) \in \mathcal{I}_{N} \times \mathcal{I}_{N}: i \neq j\right\}, \quad \forall v \in \mathcal{V}(b)$,

where

$$
\begin{aligned}
& \Upsilon_{i j}^{[1]}(g):=\left[\begin{array}{ccc}
-0.5 P_{i} & * & * \\
0 & -0.5 \varepsilon_{1}^{-1}\left(1+\lambda \varepsilon_{2}\right) I_{q} & * \\
g \mathcal{T}\left(A_{i} P_{j}+B_{i} F_{j}-P_{i}\right) & g \mathcal{T} B_{w, i} & -2 b+\left(1+\lambda \varepsilon_{2}\right)
\end{array}\right], \\
& \Upsilon_{i j k}^{[2]}:=\left[\begin{array}{ccc}
-0.5 P_{i} & * & * \\
0 & -0.5 \varepsilon_{1}^{-1}\left(1+\lambda \varepsilon_{2}\right) I_{q} & * \\
\mathcal{T}_{k}\left(A_{i} P_{j}+B_{i} F_{j}\right) & \mathcal{T}_{k} B_{w, i} & -2 z_{l, \max }+\left(1+\lambda \varepsilon_{2}\right)
\end{array}\right], \\
& \Upsilon_{i j}^{[3]}(v):=\left[\begin{array}{ccc}
-P_{i} & * & * \\
0 & -\lambda I_{q} & * \\
A_{i} P_{j}+B_{i} F_{j} & B_{w, i} & -P_{i}-\sum_{k=1}^{N}\left(P_{k}+M_{i j}\right) v_{k}
\end{array}\right] .
\end{aligned}
$$

The following theorem is the main result of this paper, where it is established that if Problem 2 is feasible, than (2) is locally asymptotically stabilizable, and a stabilizing controller can be constructed from the solution of Problem 2.

Theorem 1. Suppose that the set of matrices $P_{i}=P_{i}^{T} \in \mathbb{R}^{n \times n}, F_{i} \in \mathbb{R}^{m \times n}, M_{i j}=M_{i j}^{T} \in \mathbb{R}^{n \times n},(i, j) \in \mathcal{I}_{N} \times \mathcal{I}_{N}$, and scalars $\beta, \lambda$ is an optimal solution to Problem 2. Then, for the closed-loop system (7), the following statements hold true:

1. Under Assumption 3, if $x(0) \in \Omega(1)$, then all the future trajectories will remain within modelling region $\mathcal{L}$;

2. Moreover, the closed-loop system (7) is locally asymptotically stable, and an invariant subset of the DA for the closed-loop system is given by $\Omega(1)$;

3. By minimizing $\beta>0$ subject to LMIs (8)-(12), the levelset $\Omega(1)$ is enlarged;

4. If $w(k) \equiv 0_{q}$, then an invariant subset of the DA for the closed-loop system is given by $\Omega\left(1+\lambda \varepsilon_{2}\right)$. 
Remark 2. Note that in (9), since $\mathcal{G}_{k}$ is a finite set of the vertices, (9) is a finite set of LMIs.

Proof of Theorem 1. To begin with, note that using Lemma 1, Lemma 3, relations $\partial \alpha_{i}(\mathcal{T} \xi) / \partial \mathcal{T} \xi \in \operatorname{co}\left\{\mathcal{G}_{i}\right\}, \forall \xi \in$ $\mathcal{L}, \forall i \in \mathcal{I}_{N}$ and $\sum_{i=1}^{N} \Delta \alpha_{i}(z(k))=0$, LMIs (8)-(12) ensure

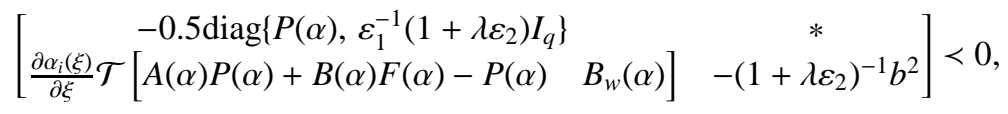

$$
\begin{aligned}
& \forall(x(k), i) \in \mathcal{L} \times \mathcal{I}_{N}, \quad \forall \xi \in\{\mathcal{T} y: y \in \mathcal{L}\}, \\
& {\left[\begin{array}{cc}
-P(\alpha) & * \\
\mathcal{T}_{i} P(\alpha) & -\left(1+\lambda \varepsilon_{2}\right)^{-1} z_{i, \max }^{2}
\end{array}\right] \prec 0, \quad \forall(x(k), i) \in \mathcal{L} \times \mathcal{I}_{p}}
\end{aligned}
$$

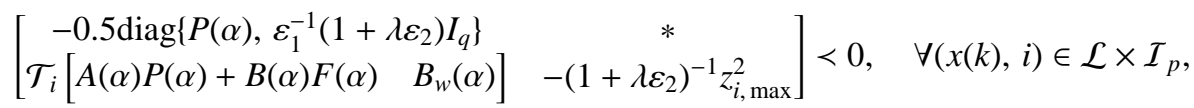

$$
\begin{aligned}
& {\left[\begin{array}{cc}
-\beta I_{n} & I_{n} \\
I_{n} & -P(\alpha)
\end{array}\right] \prec 0, \quad \forall x(k) \in \mathcal{L}}
\end{aligned}
$$

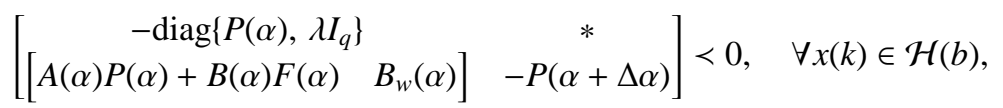

Note that since (17) is derived from LMIs in (12) based on assumption $\left|\alpha_{i}(z(k))\right| \leq b, \forall i \in \mathcal{I}_{N},(17)$ is satisfied only when $x(k) \in \mathcal{H}(b) \subseteq \mathcal{L}$, where $\mathcal{H}(b)$ is the set of state variables in which $\left|\alpha_{i}(z(k))\right| \leq b$ is satisfied for all $i \in \mathcal{I}_{N}$. To prove statements 1$)-3$ ), we need to prove the followings:

a) $\Omega\left(1+\lambda \varepsilon_{2}\right) \subset \mathcal{L}$;

b) $\Omega\left(1+\lambda \varepsilon_{2}\right) \subseteq \mathcal{R}$;

c) $\Omega\left(1+\lambda \varepsilon_{2}\right) \subseteq \mathcal{H}(b)$;

d) $\left\{x: x^{T} x \leq 1 / \beta\right\} \cap \mathcal{L} \subseteq \Omega(1)$

where $\mathcal{H}(b):=\left\{x \in \mathcal{L}:\left|\Delta \alpha_{i}(z)\right| \leq b, z=\mathcal{T} x, \forall i \in \mathcal{I}_{N}\right\}$. The inclusion relations among the sets $\mathcal{L}, \mathcal{R}, \mathcal{H}(b)$, $\Omega\left(1+\lambda \varepsilon_{2}\right)$, and $\Omega(1)$ are shown in Figure 1 .

Proof for statement a): Applying the congruence transformation to (14) with $\operatorname{diag}\left\{P(\alpha)^{-1}, 1\right\}$, using the Schur complement, and multiplying both sides of the resulting inequality by $\left(1+\lambda \varepsilon_{2}\right)^{-1}$, we have

$$
\begin{aligned}
& z_{i, \max }^{-2} \mathcal{T}_{i}^{T} \mathcal{T}_{i}<\left(1+\lambda \varepsilon_{2}\right)^{-1} P(\alpha)^{-1}, \quad \forall(x(k), i) \in \mathcal{L} \times \mathcal{I}_{p} \\
& \Leftrightarrow z_{i, \max }^{-2} z_{i}(k)^{2}<\left(1+\lambda \varepsilon_{2}\right)^{-1} x(k)^{T} P(\alpha)^{-1} x(k), \quad \forall(x(k), i) \in \mathcal{L} \backslash\left\{0_{n}\right\} \times \mathcal{I}_{p} \\
& \Rightarrow 1+\lambda \varepsilon_{2}<x(k)^{T} P(\alpha)^{-1} x(k), \quad \forall x(k) \in \partial \mathcal{L},
\end{aligned}
$$

which implies $1+\lambda \varepsilon_{2}<\sigma:=\min _{x \in \partial \mathcal{L}} x^{T} P(\alpha)^{-1} x$. Since $\Omega\left(1+\lambda \varepsilon_{2}\right) \subset \Omega(\sigma) \subseteq \mathcal{L}$, we have $\Omega\left(1+\lambda \varepsilon_{2}\right) \subset \mathcal{L}$.

Proof for statement $\mathbf{b}$ ): Applying the congruence transformation to (15) with $\operatorname{diag}\left\{P(\alpha)^{-1}, I_{q}, 1\right\}$, using the Schur complement, and multiplying both sides of the resulting inequality by $\left(1+\lambda \varepsilon_{2}\right)^{-1}$, we have

$$
-0.5\left[\begin{array}{cc}
\left(1+\lambda \varepsilon_{2}\right)^{-1} P(\alpha)^{-1} & 0_{n \times q} \\
0_{q \times n} & \varepsilon_{1}^{-1} I_{q}
\end{array}\right]+z_{i, \max }^{-2}\left[A_{c l}(\alpha) \quad B_{w}(\alpha)\right]^{T} \mathcal{T}_{i}^{T} \mathcal{T}_{i}\left[A_{c l}(\alpha) \quad B_{w}(\alpha)\right] \prec 0, \quad \forall(x(k), i) \in \mathcal{L} \times \mathcal{I}_{p},
$$

where $A_{c l}(\alpha):=A(\alpha)+B(\alpha) F(\alpha) P(\alpha)^{-1}$. Pre- and post-multiplying the last inequality by $\left[x(k)^{T} \quad w(k)^{T}\right]$ and its transpose yields

$$
\begin{aligned}
& z_{i, \max }^{-2}\left(A_{c l}(\alpha) x(k)+B_{w}(\alpha) w(k)\right)^{T} \mathcal{T}_{i}^{T} \mathcal{T}_{i}\left(A_{c l}(\alpha) x(k)+B_{w}(\alpha) w(k)\right) \\
& =z_{i, \max }^{-2} x(k+1)^{T} \mathcal{T}_{i}^{T} \mathcal{T}_{i} x(k+1) \\
& \leq 0.5\left(\left(1+\lambda \varepsilon_{2}\right)^{-1} x(k)^{T} P(\alpha)^{-1} x(k)+\varepsilon_{1}^{-1} w(k)^{T} w(k)\right), \quad \forall(x(k), i) \in \mathcal{L} \times \mathcal{I}_{p} \\
& 7
\end{aligned}
$$




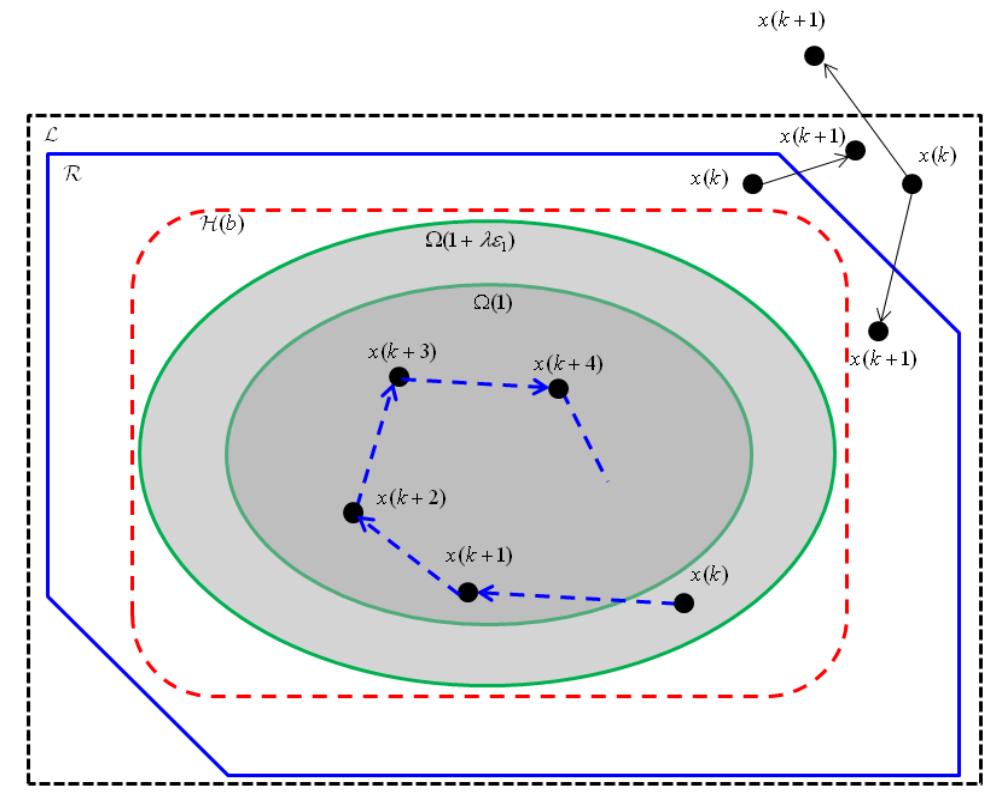

Figure 1: Inclusion relations among the sets $\mathcal{L}, \mathcal{R}, \mathcal{H}(b), \Omega\left(1+\lambda \varepsilon_{2}\right)$, and $\Omega(1)$.

$$
\leq 0.5(1+1)=1, \quad \forall(x(k), i) \in\left(\mathcal{L} \cap \Omega\left(1+\lambda \varepsilon_{2}\right)\right) \times \mathcal{I}_{p},
$$

where we used $w(k)^{T} w(k) \leq \varepsilon_{1}, \forall k \in \mathbb{Z}_{+}$in Assumption 3. Again, the last inequality can be rewritten as

$$
z_{i}^{2}(k+1) \leq z_{i, \max }^{2}, \quad \forall(x(k), i) \in\left(\mathcal{L} \cap \Omega\left(1+\lambda \varepsilon_{2}\right)\right) \times \mathcal{I}_{p} .
$$

From Statement a), we know $\Omega\left(1+\lambda \varepsilon_{2}\right) \subset \mathcal{L}$, so it follows that

$$
z_{i}^{2}(k+1) \leq z_{i, \max }^{2}, \quad \forall(x(k), i) \in \Omega\left(1+\lambda \varepsilon_{2}\right) \times \mathcal{I}_{p},
$$

which implies $x(k) \in \Omega\left(1+\lambda \varepsilon_{2}\right) \Rightarrow x(k+1) \in \mathcal{L}$. From the definition of set $\mathcal{R}$, we have $\Omega\left(1+\lambda \varepsilon_{2}\right) \subseteq \mathcal{R}$.

Proof for statement c): Applying the congruence transformation to (13) with $\operatorname{diag}\left\{P(\alpha)^{-1}, I_{q}, 1\right\}$, using the Schur complement, and multiplying both sides of the resulting inequality by $\left(1+\lambda \varepsilon_{2}\right)^{-1}$ lead to

$$
\begin{aligned}
& -0.5 \operatorname{diag}\left\{\left(1+\lambda \varepsilon_{2}\right)^{-1} P(\alpha)^{-1}, \varepsilon_{1}^{-1} I_{q}\right\}+b^{-2}\left[A_{c l}(\alpha)-I_{n} \quad B_{w}(\alpha)\right]^{T} \mathcal{T}^{T}\left[\frac{\partial \alpha_{i}(\xi)}{\partial \xi}\right]^{T}\left[\frac{\partial \alpha_{i}(\xi)}{\partial \xi}\right] \mathcal{T}\left[A_{c l}(\alpha)-I_{n} \quad B_{w}(\alpha)\right]<0, \\
& \forall(x(k), i) \in \mathcal{R} \times \mathcal{I}_{N}, \quad \forall \xi \in\{\mathcal{T} y: y \in \mathcal{L}\} .
\end{aligned}
$$

Since $\mathcal{R} \subseteq \mathcal{L}$ from the definition of $\mathcal{R}$, the last inequality holds for all $x(k) \in \mathcal{R}$. Pre- and post-multiplying the last inequality by $\left[x(k)^{T} \quad w(k)^{T}\right]$ and its transpose yields

$$
\begin{aligned}
& b^{-2}\left[\begin{array}{c}
x(k) \\
w(k)
\end{array}\right]^{T}\left[\begin{array}{ll}
A_{c l}(\alpha)-I_{n} & B_{w}(\alpha)
\end{array}\right]^{T} \mathcal{T}^{T}\left[\frac{\partial \alpha_{i}(\xi)}{\partial \xi}\right]^{T}\left[\frac{\partial \alpha_{i}(\xi)}{\partial \xi}\right] \mathcal{T}\left[A_{c l}(\alpha)-I_{n} \quad B_{w}(\alpha)\right]\left[\begin{array}{c}
x(k) \\
w(k)
\end{array}\right] \\
& \leq 0.5\left(\left(1+\lambda \varepsilon_{2}\right)^{-1} x(k)^{T} P(\alpha)^{-1} x(k)+\varepsilon_{1}^{-1} w(k)^{T} w(k)\right), \quad \forall(x(k), i) \in \mathcal{R} \times \mathcal{I}_{N}, \quad \forall \xi \in\{\mathcal{T} y: y \in \mathcal{L}\} .
\end{aligned}
$$

Next, we need to consider the following argument: If $x(k) \in \mathcal{R}$, then by the definition of set $\mathcal{R}$, both $x(k)$ and $x(k+1)$ lie in $\mathcal{L}$. Since $\mathcal{L}$ is a convex set in $\mathbb{R}^{n},(1-c) x(k+1)+c x(k) \in \mathcal{L}$ holds for any $c \in[0,1]$. In addition, we have $(1-c) \mathcal{T} x(k+1)+c \mathcal{T} x(k)=(1-c) z(k+1)+c z(k) \in\left[-z_{1, \max }, z_{1, \max }\right] \times \cdots \times\left[-z_{p, \max }, z_{p, \max }\right]$. Therefore, by the mean value theorem in several variables (Lemma 2$)$, there are some real numbers $\left(c_{1}, c_{2}, \ldots, c_{N}\right) \in[0,1]^{N}$ for each sample $k$ such that

$$
\Delta \alpha_{i}(z(k))=\frac{\partial \alpha_{i}\left(\left(1-c_{i}\right) z(k+1)+c_{i} z(k)\right)}{\partial z(k)} \mathcal{T}(x(k+1)-x(k)), \quad \forall(x(k), i) \in \mathcal{R} \times \mathcal{I}_{N} .
$$


This means that, there always exists $g_{i}(k) \in \operatorname{co}\left\{\mathcal{G}_{i}\right\}$ for each sample $k$ such that

$$
\begin{aligned}
& \Delta \alpha_{i}(z(k))=g_{i}(k) \mathcal{T}(x(k+1)-x(k)), \quad \forall(x(k), i) \in \mathcal{R} \times \mathcal{I}_{N} \\
& \Leftrightarrow \Delta \alpha_{i}(z(k))=g_{i}(k) \mathcal{T}\left[A_{c l}(\alpha)-I_{n} \quad B_{w}(\alpha)\right]\left[\begin{array}{c}
x(k) \\
w(k)
\end{array}\right], \quad \forall(x(k), i) \in \mathcal{R} \times \mathcal{I}_{N} .
\end{aligned}
$$

Plugging the above equation into (18) yields

$$
b^{-2} \Delta \alpha_{i}(z(k))^{2} \leq 0.5\left(\left(1+\lambda \varepsilon_{2}\right)^{-1} x(k)^{T} P(\alpha) x(k)+\varepsilon_{1}^{-1} w(k)^{T} w(k)\right), \quad \forall(x(k), i) \in \mathcal{R} \times \mathcal{I}_{N} .
$$

In addition, $w(k)^{T} w(k) \leq \varepsilon_{1}, \forall k \in \mathbb{Z}_{+}$in Assumption 3 results in

$$
\begin{aligned}
b^{-2} \Delta \alpha_{i}(z(k))^{2} & \leq 0.5\left(\left(1+\lambda \varepsilon_{2}\right)^{-1} x(k)^{T} P(\alpha) x(k)+\varepsilon_{1}^{-1} w(k)^{T} w(k)\right) \\
& \leq 0.5\left(\left(1+\lambda \varepsilon_{2}\right)^{-1} x(k)^{T} P(\alpha) x(k)+1\right) \\
& \leq 0.5(1+1) \\
& =1, \quad \forall(x(k), i) \in\left(\mathcal{R} \cap \Omega\left(1+\lambda \varepsilon_{2}\right)\right) \times \mathcal{I}_{N}
\end{aligned}
$$

From $\Omega\left(1+\lambda \varepsilon_{2}\right) \subseteq \mathcal{R}$ of statement b), it follows that $\Delta \alpha_{i}(z(k))^{2} \leq b^{2}, \forall(x(k), i) \in \Omega\left(1+\lambda \varepsilon_{2}\right) \times \mathcal{I}_{N}$, from which one has $\Omega\left(1+\lambda \varepsilon_{2}\right) \subseteq \mathcal{H}(b)$.

Proof of statement d): We apply the Schur complement to (16) to obtain

$$
\begin{aligned}
& P(\alpha)^{-1}<\beta I_{n}, \quad \forall x(k) \in \mathcal{L} \\
& \Rightarrow V(x(k)) \leq \beta x(k)^{T} x(k), \quad \forall x(k) \in \mathcal{L} \\
& \Rightarrow V(x(k))-1 \leq \beta x(k)^{T} x(k)-1, \quad \forall x(k) \in \mathcal{L} \\
& \Rightarrow V(x(k))-1 \leq \beta\left(x(k)^{T} x(k)-1 / \beta\right), \quad \forall x(k) \in \mathcal{L} \\
& \Rightarrow\left\{x \in \mathbb{R}^{n}: x^{T} x \leq 1 / \beta\right\} \cap \mathcal{L} \subseteq \Omega(1) \cap \mathcal{L}
\end{aligned}
$$

Since $\Omega\left(1+\lambda \varepsilon_{2}\right) \subset \mathcal{L}$ from statement a), we have $\Omega(1) \cap \mathcal{L}=\Omega(1)$, and hence $\left\{x: x^{T} x \leq 1 / \beta\right\} \cap \mathcal{L} \subseteq \Omega(1)$.

Next, using statements a)-d), we can prove statement 1 ) as follows:

Proof for statement 1): Applying the congruence transformation to (17) with $\operatorname{diag}\left\{P(\alpha)^{-1}, I_{q}, I_{n}\right\}$ and using the Schur complement, one gets

$$
\left[\begin{array}{ll}
A_{c l}(\alpha) & B_{w}(\alpha)
\end{array}\right]^{T} P(\alpha+\Delta \alpha)^{-1}\left[A_{c l}(\alpha) \quad B_{w}(\alpha)\right]-\left[\begin{array}{cc}
P(\alpha)^{-1} & 0_{n \times q} \\
0_{q \times n} & \lambda I_{q}
\end{array}\right] \prec 0, \quad \forall x(k) \in \mathcal{H}(b),
$$

which leads to

$$
\begin{aligned}
& {\left[\begin{array}{c}
x(k) \\
w(k)
\end{array}\right]^{T}\left[\begin{array}{ll}
A_{c l}(\alpha) & B_{w}(\alpha)
\end{array}\right]^{T} P\left((\alpha+\Delta \alpha)^{-1}\left[\begin{array}{ll}
A_{c l}(\alpha) & B_{w}(\alpha)
\end{array}\right]\left[\begin{array}{c}
x(k) \\
w(k)
\end{array}\right]-x(k)^{T} P(\alpha)^{-1} x(k)-\lambda w(k)^{T} w(k)\right.} \\
& =V(x(k+1))-V(x(k))-\lambda w(k)^{T} w(k)<0, \quad \forall x(k) \in \mathcal{H}(b) \backslash\left\{0_{n}\right\} .
\end{aligned}
$$

The next step is to sum the left-hand side of (20) from $k=0$ to an arbitrary time $T$. However, there is no guarantee that state $x(k)$ does not escape set $\mathcal{H}(b)$ during time interval $k \in[0, T]$. In this case, the inequality (20) cannot be summed since it holds only when $x(k) \in \mathcal{H}(b) \backslash\left\{0_{n}\right\}$. We will proof that this case does not occur. Suppose that the last inequality holds but $x(k)$ starting from $x(0) \in \Omega(1)$ satisfies

$$
\left\{\begin{array}{l}
x(k) \in \Omega\left(1+\lambda \varepsilon_{2}\right), \quad k \in\{0,1, \ldots, T-1\} ; \\
x(T) \in \Omega\left(1+\lambda \varepsilon_{2}\right)^{C} .
\end{array}\right.
$$

for some $T \in \mathbb{N}_{+}$. From the statement 2), $\Omega\left(1+\lambda \varepsilon_{2}\right) \subseteq \mathcal{H}(b)$ holds. Therefore, $x(k) \in \mathcal{H}(b)$ holds for all $k \in$ $\{0,1, \ldots, T-1\}$. This implies that $(20)$ is satisfied for $k \in\{0,1, \ldots, T-1\}$. Therefore, we can sum the left-hand side of (20) from 0 to $T-1$ in order to obtain

$$
\sum_{k=0}^{T-1}[V(x(k+1))-V(x(k))]=V(x(T))-V(x(0))<\lambda \sum_{k=0}^{T-1} w(k)^{T} w(k)
$$




$$
\Rightarrow V(x(T))<V(x(0))+\lambda \sum_{k=0}^{T-1} w(k)^{T} w(k) \leq 1+\lambda \varepsilon_{2} .
$$

Hence, $V(x(T))<1+\lambda \varepsilon_{2} \Rightarrow x(T) \in \Omega\left(1+\lambda \varepsilon_{2}\right)$, which gives a contradiction. This implies that the state $x(k)$ will not reach the boundary of $\Omega\left(1+\lambda \varepsilon_{2}\right)$. In other words, $x(k)$ starting from $x(0) \in \Omega(1)$ does not escape domain $\Omega\left(1+\lambda \varepsilon_{2}\right)$. Since $\Omega\left(1+\lambda \varepsilon_{2}\right) \subset \mathcal{L}$ from statement a), one concludes that $x(k)$ starting from $x(0) \in \Omega(1)$ does not escape modelling region $\mathcal{L}$.

Proof for statement 2): The inequality (19) ensures the existence of a real number $c>0$ such that $V(x(k+1))-$ $V(x(k))-\lambda w(k)^{T} w(k)<-c x(k)^{T} x(k)-c w(k)^{T} w(k), \forall x(k) \in \mathcal{H}(b) \backslash\left\{0_{n}\right\}$, which can be rewritten as

$$
V(x(k+1))-V(x(k))<-c x(k)^{T} x(k)+(\lambda-c) w(k)^{T} w(k), \quad \forall x(k) \in \mathcal{H}(b) \backslash\left\{0_{n}\right\} .
$$

From the statement 1), it can be proved that if $x(0) \in \Omega(1)$ and $x(k) \neq 0_{n}$, then the above inequality holds for all $k \in \mathbb{Z}_{+}$. Assume that the closed-loop system (2) is not asymptotically stable., which implies

$$
\sum_{k=0}^{\infty} x(k)^{T} x(k)=\infty
$$

Summing both sides of (21) from $k=0$ to $k=\infty$, we have

$$
c \sum_{k=0}^{\infty} x(k)^{T} x(k)<V(x(0))+|\lambda-c| \varepsilon_{2}, \quad \forall x(k) \in \mathcal{H}(b) \backslash\left\{0_{n}\right\} .
$$

This ensures that the left-hand side is bounded. This contradicts with the hypothesis. Therefore, the closed-loop system (2) is asymptotically stable.

Proof for statement 3): Minimizing $\beta$ while imposing constraint $\left\{x \in \mathbb{R}^{n}: x^{T} x \leq 1 / \beta\right\} \cap \mathcal{L} \subseteq \Omega(1)$ makes $\Omega(1)$ to be enlarged.

Proof for statement 4): Suppose $w(k) \equiv 0_{q}$. In this case, inequality (20) becomes

$$
V(x(k+1))-V(x(k))<0, \quad \forall x(k) \in \mathcal{H}(b) \backslash\left\{0_{n}\right\},
$$

which implies

$$
\mathcal{H}(b) \backslash\left\{0_{n}\right\} \subseteq\left\{x \in \mathcal{L} \backslash\left\{0_{n}\right\}: V(x(k+1))-V(x(k))<0\right\} .
$$

On the other hand, from statement c), one has $\Omega\left(1+\lambda \varepsilon_{2}\right) \subset \mathcal{H}(b)$. Thus, (22) implies $\Omega\left(1+\eta \varepsilon_{2}\right) \backslash\left\{0_{n}\right\} \subseteq\left\{x \in \mathcal{L} \backslash\left\{0_{n}\right\}\right.$ : $V\left(A_{c l}(\alpha) x\right)-V(x)<0$. By the Lyapunov theory [14], (2) is locally asymptotically stable, and $\Omega\left(1+\lambda \varepsilon_{2}\right)$ is an invariant subset of the DA [14].

Remark 3. In Theorem 1, some design parameters should be chosen by the designer. The parameter $\beta>0$ is automatically determined by solving Problem 2 . The parameters $\varepsilon_{1}>0$ and $\varepsilon_{2}>0$ can be pre-determined based on the disturbance modelling. On the other hand, $b \in(0,1]$ should be selected by the designer before solving Problem 2. Unfortunately, there is no general guideline on how to select $b$ to find a feasible solution and improve the performance. At the current stage, it can be determined by a combination of previous expertise and trial and error. Typically, the smaller the bound $b$, the less conservative the condition of Problem 2. On the other hand, if $b$ is too small, due to the constraint $\Omega\left(1+\lambda \varepsilon_{2}\right) \subseteq \mathcal{H}(b)$, the volume of set can also be reduced. The same issue was discussed in [18, Remark 3]. Similarly to [18, Remark 3], one of strategies to determine $b$ is to initially select it small enough and if Problem 2 is feasible for the value of $b$, then one can increase $b$ until it becomes infeasible. Motivated by this discussion, the following control design procedure can be suggested:

1. Given a discrete-time nonlinear system $x(k+1)=f(x(k), u(k), w(k))$, calculate the subsystem matrices $\left(A_{i}, B_{i}, B_{w i}\right), i \in$ $\mathcal{I}_{N}$, the membership functions $\alpha_{i}(z(k)), i \in \mathcal{I}_{N}$, the premise variables $z(k)=\mathcal{T} x(k)$, and the modelling region $\mathcal{L}$ for the T-S fuzzy model (2); 
2. For each $i \in \mathcal{I}_{N}$, calculate $\partial \alpha_{i}(\xi) / \partial \xi$ and the set of vertices $\mathcal{G}_{i}$ that includes $\partial \alpha_{i}(\xi) / \partial \xi$ for all $\xi \in \mathcal{T} x, x \in \mathcal{L}$;

3. Determine the parameters $\varepsilon_{1}>0$ and $\varepsilon_{2}>0$ based on the disturbance model;

4. Choose a sufficiently small $b \in(0,1]$ and solve the optimization in Problem 2;

5. If, infeasible, then the system (2) cannot be identified as locally asymptotically stable via the optimization of Problem 2. Otherwise, if feasible, then the system (2) is locally asymptotically stable, and the statements 1-4 in Theorem 1 hold;

6. Compute the maximum $b \in(0,1]$ such that the optimization in Problem 2 is feasible. A simple line search or the bisection algorithm can be applied.

Remark 4. A general $\gamma$-level set $\Omega(\gamma)$ can be used as an estimation of the DA instead of the 1-level set $\Omega(1)$ in Theorem 1. However, for any given $\gamma, \Omega(\gamma)$ can be converted to $\Omega(1)$ with the scaling of the Lyapunov matrices $P_{i}$, i.e., $P_{i} / \gamma, \forall i \in \mathcal{I}_{N}$, and the feasibility of the Problem 2 and the computed estimation of the DA will not be changed for different values of $\gamma$.

\section{Examples}

The LMI problems in the sequel were solved with SeDuMi [33] and Yalmip [22].

Example 2. Consider the system (2) with

$$
A_{1}=\left[\begin{array}{cc}
1 & -1.2 \\
-1 & -0.5
\end{array}\right], \quad A_{2}=\left[\begin{array}{cc}
1 & 1.2 \\
-1 & -0.5
\end{array}\right], \quad B_{1}=\left[\begin{array}{l}
6.2 \\
2.4
\end{array}\right], \quad B_{2}=\left[\begin{array}{c}
3.8 \\
-2.4
\end{array}\right], \quad B_{w 1}=\left[\begin{array}{c}
0.1 \\
1
\end{array}\right], \quad B_{w 2}=\left[\begin{array}{c}
0.1 \\
1
\end{array}\right],
$$

and the membership functions $\alpha_{1}(z(k))=\left(1+\sin x_{1}(k)\right) / 2, \alpha_{2}(z(k))=1-\alpha_{1}(z(k))$ defined in $\mathcal{L}=\left\{x \in \mathbb{R}^{n}: \mathcal{T} x=\right.$ $\left.x_{1} \in[-\pi / 2, \pi / 2]\right\}$, where $\mathcal{T}=\left[\begin{array}{ll}1 & 0\end{array}\right]$. Since

$$
\frac{\partial \alpha_{1}(z(k))}{\partial z(k)}=0.5 \cos x_{1}(k) \in \operatorname{co}\{0,0.5\}, \quad \frac{\partial \alpha_{2}(z(k))}{\partial z(k)}=-0.5 \cos x_{1}(k) \in \operatorname{co}\{0,-0.5\},
$$

for all $x(k) \in \mathcal{L}$, we have $\mathcal{G}_{1}=\{0,0.5\}$ and $\mathcal{G}_{2}=\{0,-0.5\}$. Under Assumption 3 with $\varepsilon_{1}=1$ and $\varepsilon_{2}=30$, the stability is evaluated using the proposed Theorem 1 with $b=1.4355$. It can be obtained based on the search procedure explained in Remark 3. The simulation result is depicted in Figure 2, which shows converging trajectories (solid lines with bullet) initialized at the " $\triangle$ " marks, level sets $\Omega(1)$ (red solid line) and $\Omega\left(1+\lambda \varepsilon_{2}\right)$ (blue dashed line) estimated by using Theorem 1 , and modelling region $\mathcal{L}$ (shaded area). For this simulation, we used the following disturbance signal:

$$
\left\{\begin{array}{cc}
w(k)=(-1)^{k} \sqrt{\varepsilon_{1}}, & \text { if } 0 \leq k \leq 30 \\
w(k)=0, & \text { if } k>30
\end{array}\right.
$$

which satisfies $w(k)^{T} w(k) \leq \varepsilon_{1}=1, \forall k \in \mathbb{Z}_{+}$and $\sum_{0}^{\infty} w(k)^{T} w(k) \leq \varepsilon_{2}=30$. The result shows that the states starting from domain $\Omega(1)$ do not escape $\mathcal{L}$ as desired.

Example 3. Consider the chaotic Lorenz system

$$
\left[\begin{array}{c}
\dot{x}_{1}(t) \\
\dot{x}_{2}(t) \\
\dot{x}_{3}(t)
\end{array}\right]=\left[\begin{array}{c}
-\sigma x_{1}(t)+\sigma x_{2}(t) \\
\rho x_{1}(t)-x_{2}(t)-x_{1}(t) x_{3}(t) \\
-\varepsilon x_{3}(t)+x_{1}(t) x_{2}(t)
\end{array}\right]
$$

which has only one equilibrium point at the origin when $\rho<1$. It can be approximately represented by a discretized T-S fuzzy system (2) with

$$
A_{1}=I_{3}+T_{s}\left[\begin{array}{ccc}
-\sigma & \sigma & 0 \\
\rho & -1 & z_{1, \max } \\
0 & -z_{1, \max } & -\varepsilon
\end{array}\right], \quad A_{2}=I_{3}+T_{s}\left[\begin{array}{ccc}
-\sigma & \sigma & 0 \\
\rho & -1 & -z_{1, \max } \\
0 & z_{1, \max } & -\varepsilon
\end{array}\right]
$$




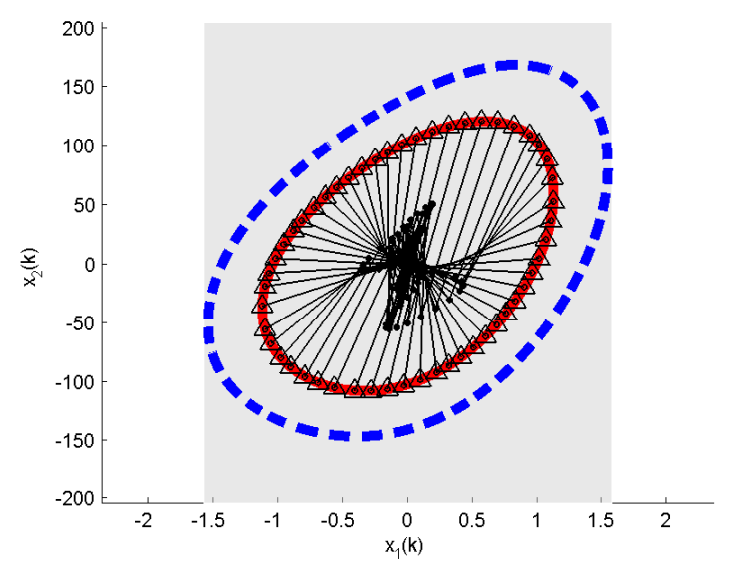

Figure 2: Example 2. Converging trajectories (solid lines with bullet) initialized at the " $\Delta$ " marks, level sets $\Omega(1)$ (red solid line) and $\Omega\left(1+\lambda \varepsilon_{2}\right)$ (blue dashed line) estimated by using Theorem 1 , and modelling region $\mathcal{L}$ (shaded area).

$$
\begin{aligned}
& z(k)=z_{1}(k)=\mathcal{T}_{1} x(k)=x_{1}(k), \quad \mathcal{T}=\mathcal{T}_{1}=\left[\begin{array}{lll}
1 & 0 & 0
\end{array}\right], \\
& \alpha_{1}(z(k))=\frac{-x_{1}(k)+z_{1, \max }}{2 z_{1, \max }}, \quad \alpha_{2}(z(k))=\frac{x_{1}(k)+z_{1, \max }}{2 z_{1, \max }}, \\
& \mathcal{L}=\left\{x \in \mathbb{R}^{n}:\left[\begin{array}{lll}
1 & 0 & 0
\end{array}\right] x \in\left[-z_{1, \max }, z_{1, \max }\right]\right\} .
\end{aligned}
$$

In this case,

$$
\frac{\partial \alpha_{1}(z(k))}{\partial x_{1}(k)}=\frac{-1}{2 z_{1, \max }} \in \operatorname{co}\left\{\frac{-1}{2 z_{1, \max }}\right\}, \quad \forall x(k) \in \mathcal{L}, \quad \frac{\partial \alpha_{2}(z(k))}{\partial x_{1}(k)}=\frac{1}{2 z_{1, \max }} \in \operatorname{co}\left\{\frac{1}{2 z_{1, \max }}\right\}, \quad \forall x(k) \in \mathcal{L},
$$

and hence, we have $\mathcal{G}_{1}=\left\{-1 / 2 z_{1, \max }\right\}$ and $\mathcal{G}_{2}=\left\{1 / 2 z_{1, \max }\right\}$. For the simulation, suppose $\left(\sigma, \rho, \varepsilon, T_{s}\right)=(10,28,8 / 3,0.002)$, $\varepsilon_{1}=1, \varepsilon_{2}=30, z_{1, \max }=50$,

$$
B_{1}=B_{2}=T_{s}\left[\begin{array}{l}
1 \\
1 \\
0
\end{array}\right], \quad B_{w 1}=B_{w 2}=\left[\begin{array}{l}
1 \\
1 \\
1
\end{array}\right],
$$

and the same disturbance input as in Example 2. Figure 3 illustrates the surfaces of the Lyapunov sublevel sets $\Omega(1)$ (red inner sphere) and $\Omega\left(1+\lambda \varepsilon_{2}\right)$ (blue outer sphere) obtained by using Theorem 1 with $b=199.9939$, where the solid line with bullet is a converging trajectory initialized at the " $\Delta$ " mark.

Example 4. Consider the fuzzy model of the truck-trailer model (5) in Example 1 again. In this example, the model parameters are chosen to be

$$
\left(l, L, v, T_{s}, z_{1, \max }\right)=\left(10[\mathrm{~m}], 3[\mathrm{~m}],-1[\mathrm{~m} / \mathrm{s}], 0.01[\mathrm{~s}], \frac{179.4270}{180} \pi[\mathrm{rad}]\right) .
$$

To apply the proposed method, define

$$
\frac{\partial \alpha_{1}(z(k))}{\partial z_{1}(k)}=\frac{z_{1, \max }}{z_{1, \max }-\sin z_{1, \max }}\left(\frac{\cos z_{1}(k)}{z_{1}(k)}-\frac{\sin z_{1}(k)}{z_{1}(k)^{2}}\right)=: f\left(z_{1}(k)\right) .
$$

Then, we have

$$
\frac{\partial \alpha_{2}(z(k))}{\partial z_{1}(k)}=\frac{\partial}{\partial z_{1}(k)}\left(1-\alpha_{1}(z(k))\right)=-\frac{\partial \alpha_{1}(z(k))}{\partial z_{1}(k)}=-f\left(z_{1}(k)\right) .
$$






Figure 3: Example 3. Converging trajectory (solid line with bullet) initialized at the " $\Delta$ " mark, level sets $\Omega(1)$ (red inner sphere) and $\Omega\left(1+\lambda \varepsilon_{2}\right)$ (blue outer sphere) estimated by using Theorem 1.



Figure 4: Example 4. Converging trajectory (solid line with bullet) initialized at the " $\Delta$ " mark, level sets $\Omega(1)$ (red inner sphere) and $\Omega\left(1+\lambda \varepsilon_{2}\right)$ (blue outer sphere) estimated by using Theorem 1.

Since $\max _{z_{1}(k) \in\left[-z_{1, \max }, z_{1, \max }\right]} f\left(z_{1}(k)\right) \cong 0.4376, \min _{z_{1}(k) \in\left[-z_{1, \max }, z_{1, \max }\right]} f\left(z_{1}(k)\right) \cong-0.4376$, one concludes that $\frac{\partial \alpha_{1}(z(k))}{\partial z_{1}(k)} \in$ $\operatorname{co}\{-0.4376,0.4376\}, \frac{\partial \alpha_{2}(z(k))}{\partial z_{1}(k)} \in \operatorname{co}\{-0.4376,0.4376\}$ hold for all $x(k) \in \mathcal{L}$. Therefore, we can set $\mathcal{G}_{1}=\mathcal{G}_{2}=$ $\{-0.4376,0.4376\}$. For $\varepsilon_{1}=30, \varepsilon_{2}=500$, and $b=4999$, the optimization in Theorem 1 is feasible and gives an optimal solution. Using the disturbance

$$
w(k)=\left\{\begin{array}{l}
(-1)^{k} \varepsilon_{1}^{1 / 2}, \quad \text { if } k \varepsilon_{1} \leq \varepsilon_{2} \\
0, \quad \text { otherwise }
\end{array}\right.
$$

the simulation result is depicted in Figure 4.

\section{Conclusion}

In this paper, a locally stabilizing controller design procedure has been developed for discrete-time T-S fuzzy systems with magnitude- and energy-bounded disturbances. The result is an extension of the continuous-time result in [42] to the discrete-time case. The differences between the proposed approach and that in [42] can be summarized 
as follows. We have adopted the recent results in $[17,18]$ on the local stability analysis. In particular, the notion of the disturbance invariant set has been newly introduced. It represents a subset of the modelling region whose next state along all the possible state trajectories does not exit the subset. Moreover, an efficient computational method to design a locally stabilizing controller has been developed. Compared to previous results, it contains less design parameters, and hence, the design procedure has been simplified. The extension of the proposed strategy to the local $H_{\infty}$ or robust control designs can be possible future research directions.

\section{References}

[1] Ahn, C. K. (2014). Receding horizon disturbance attenuation for Takagi-Sugeno fuzzy switched dynamic neural networks. Information Sciences, 280:53-63.

[2] Bernal, M. and Guerra, T. M. (2010). Generalized nonquadratic stability of continuous-time Takagi-Sugeno models. IEEE Transactoins on Fuzzy Systems, 18(4):815-822.

[3] Boyd, S., Ghaoui, L. E., Feron, E., and Balakrishnan, V. (1994). Linear Matrix Inequalities in Systems and Control Theory. Philadelphia, PA: SIAM.

[4] Buck, R. C. (1978). Advanced Calculus. McGraw-Hill, 3 edition.

[5] Chang, X.-H. and Yang, G.-H. (2014). Non-fragile $H_{\infty}$ filter design for discrete-time fuzzy systems with multiplicative gain variations. Information Sciences, 266:171-185.

[6] de Oliveira, M. C., Geromel, J. C., and Bernussou, J. (2002). Extended $H_{2}$ and $H_{\infty}$ norm characterization and controller parameterization for discrete-time systems. International Journal of Control, 75(9):666-679.

[7] Ding, B. (2010). Homogeneous polynomially nonquadratic stabilization of discrete-time Takagi-Sugeno systems via nonparallel distributed compensation law. IEEE Transactions on Fuzzy Systems, 18(5):994-1000.

[8] Feng, G. (2003). Controller synthesis of fuzzy dynamic systems based on piecewise Lyapunov functions. IEEE Transactions on Fuzzy Systems, 11(5):605-612.

[9] Guerra, T. M., Kruszewski, A., and Bernal, M. (2009). Control law proposition for the stabilization of discrete Takagi-Sugeno models. IEEE Transactions on Fuzzy Systems, 17(3):724-731.

[10] Guerra, T. M. and Vermeiren, L. (2004). LMI-based relaxed nonquadratic stabilization condttions for nonlinear systems in the TakagiSugeno's form. Automatica, 40(5):823-829.

[11] Jiang, X. (2015). On sampled-data fuzzy control design approach for T-S model-based fuzzy systems by using discretization approach. Information Sciences, 296:307-314.

[12] Johansson, M., Rantzer, A., and Arzen, M. (1999). Piecewise quadratic stability of fuzzy systems. IEEE Transactions on Fuzzy Systems, 7(6):713-722.

[13] Kerrigan, E. C. and Maciejowski, J. M. (2004). Feedback min-max model predictive control using a single linear program: Robust stability and the explicit solution. International Journal of Robust and Nonlinear Control, 14(4):395-413.

[14] Khalil, H. K. (2001). Nonlinear Systems. NJ: Prentice-Hall, Upper Saddle River, 3 edition.

[15] Kruszewski, A., Sala, A., Guerra, T. M., and no, C. A. (2009). A triangulation approach to asymptotically exact conditions for fuzzy summations. IEEE Transactions on Fuzzy Systems, 17(5):985-994.

[16] Lam, H. K. (2011). Polynomial fuzzy-model-based control systems: Stability analysis via piecewise-linear membership functions. IEEE Transactions on Fuzzy Systems, 19(3):588-593.

[17] Lee, D. H. (2013). Local stability and stabilization of discrete-time Takagi-Sugeno fuzzy systems using bounded variation rates of the membership functions. In Proceedings of IEEE Symposium Series on Computational Intelligence, Singapore, pages 57-64.

[18] Lee, D. H. and Joo, Y. H. (2014). On the generalized local stability and local stabilization conditions for discrete-time Takagi-Sugeno fuzzy systems. IEEE Transactions on Fuzzy Systems, 22(6):1654-1668.

[19] Lee, H. J. et al. (2016). Performance-recoverable intelligent digital redesign for fuzzy tracking controllers. Information Sciences, 326:350367.

[20] Lee, H. J., Park, J. B., and Chen, G. (2001). Robust fuzzy control of nonlinear systems with parametric uncertainties. IEEE Transactions on Fuzzy Systems, 9(2):369-379.

[21] Lo, J.-C. and Lin, M.-L. (2003). Robust $H_{\infty}$ nonlinear control via fuzzy static output feedback. IEEE Transactions on Circuits and Systems I: Fundamental Theory and Applications, 50(11):1494-1502.

[22] Löfberg, J. (2004). YALMIP: A toolbox for modeling and optimization in MATLAB. In Proceedings of IEEE International Symposium on Computer Aided Control Systems Design, Taipei, pages 284-289. [Online]. Available: http://control.ee.ethz.ch/ joloef/yalmip.php.

[23] Luo, M., Sun, F., and Liu, H. (2013). Hierarchical structured sparse representation for T-S fuzzy systems identification. IEEE Transactions on Fuzzy Systems, 21(6):1032-1043.

[24] Mozelli, L. A., Palhares, R. M., Souza, F. O., and Mendes, E. M. A. M. (2009). Reducing conservativeness in recent stability conditions of T-S fuzzy systems. Automatica, 45(6):1580-1583.

[25] Narimani, M. and Lam, H. K. (2009). Relaxed LMI-based stability conditions for Takagi-Sugeno fuzzy control systems using regionalmembership-function-shape-dependent analysis approach. IEEE Transactions on Fuzzy Systems, 17(5):1221-1228.

[26] Pan, J. T., Guerra, T. M., Fei, S. M., and Jaadari, A. (2012). Nonquadratic stabilization of continuous T-S fuzzy models: LMI solution for a local approach. IEEE Transactions on Fuzzy Systems, 20(3):594-602.

[27] Raković, S., Kerrigan, E. C., Kouramas, K. I., and Mayne, D. Q. (2005). Invariant approximations of the minimal robust positively invariant set. IEEE Transactions on Automatic Control, 50(3):406-410.

[28] Rhee, B. J. and Won, S. (2006). A new fuzzy Lyapunov function approach for a Takagi-Sugeno fuzzy control system design. Fuzzy Sets and Systems, 157(9):1211-1228. 
[29] Sala, A. and no, C. A. (2007a). Asymptotically necessary and sufficient conditions for stability and performance in fuzzy control: Applications of Pólya's theorem. Fuzzy Sets and Systems, 158(24):2671-2686.

[30] Sala, A. and no, C. A. (2007b). Relaxed stability and performance conditions for Takagi-Sugeno fuzzy systems with knowledge on membership function overlap. IEEE Transactions on Systems, Man, and Cybernetics-Part B: Cybernetics, 37(3):727-732.

[31] Sala, A. and no, C. A. (2009). Polynomial fuzzy models for nonlinear control: A Taylor series approach. IEEE Transactions on Fuzzy Systems, 17(6):1284-1295.

[32] Shi, P., Su, X., and Li, F. Dissipativity-based filtering for fuzzy switched systems with stochastic perturbation.

[33] Sturm, J. F. (1999). Using sedumi 1.02, a MATLAB toolbox for optimization over symmetric cones. Optimization Methods and Software, 11-12:625-653.

[34] Su, X., Shi, P., Wu, L., and Song, Y. (2015). Fault detection filtering for nonlinear switched stochastic systems. IEEE Transactions on Automatic Control, 61(5):1310-1315.

[35] Su, X., Wu, L., Shi, P., and Song, Y.-D. (2014). A novel approach to output feedback control of fuzzy stochastic systems. Automatica, 50(12):3268-3275.

[36] Tanaka, K., Hori, T., and Wang, H. O. (2001). A fuzzy Lyapunov approach to fuzzy control system design. In Proceedings of IEEE American Control Conference, Arlington, VA, USA, pages $4791-4795$.

[37] Tanaka, K., Hori, T., and Wang, H. O. (2003). A multiple Lyapunov function approach to stabilization of fuzzy control systems. IEEE Transactions on Fuzzy Systems, 11(4):582-589.

[38] Tanaka, K. and Kosaki, T. (1997). Design of a stable fuzzy controller for an articulated vehicle. IEEE Transactions on Systems, Man, and Cybernetics-Part B: Cybernetics, 27(3):552-558.

[39] Tanaka, K. and Wang, H. O. (2001). Fuzzy Control Systems Design and Analysis. Ed. New York: Wiley.

[40] Tanaka, K., Yoshida, H., Ohtake, H., and Wang, H. O. (2009). A sum-of-squares approach to modeling and control of nonlinear dynamical systems with polynomial fuzzy systems. IEEE Transactions on Fuzzy Systems, 17(4):911-922.

[41] Tuan, H. D., Apkarian, P., Narikiyo, T., and Yamamoto, Y. (2001). Parameterized linear matrix inequality techniques in fuzzy control system deisgn. IEEE Transactions on Fuzzy Systems, 9(2):324-332.

[42] Wang, L. and Liu, X. (2013). Local analysis of continuous-time Takagi-Sugeno fuzzy system with disturbances bounded by magnitude or energy: A Lagrange multiplier method. Information Science, 248(1):89-102.

[43] Xie, X., Ma, H., Zhao, Y., Ding, D. W., and Wang, Y. (2013). Control synthesis of discrete-time T-S fuzzy systems based on a novel non-PDC control scheme. IEEE Transactions on Fuzzy Systems, 21(1):147-157.

[44] Ying, H. (1998). Sufficient conditions on uniform approximation of multivariate functions by general Takagi-Sugeno fuzzy systems with linear rule consequent. IEEE Transactions on Systems, Man, and Cybernetics-Part A: Systems and Humans, 28(4):515-520. 